\title{
Plant invasion alters latitudinal pattern of plant-defense syndromes
}

\author{
Mu Liu, ${ }^{1}$ Yuanfei Pan, ${ }^{1,2}$ Xiaoyun Pan (iD $, 1,3,4,10$ Alejandro Sosa,,${ }^{5,6}$ Dana M. Blumenthal, ${ }^{7}$ \\ Mark Van Kleunen (iD, ${ }^{8,9}$ and Bo Li $^{1}$ \\ ${ }^{1}$ Ministry of Education Key Laboratory for Biodiversity Science and Ecological Engineering, National Observations and Research \\ Station for Wetland Ecosystems of the Yangtze Estuary, Institute of Biodiversity Science and Institute of Eco-Chongming, School of \\ Life Sciences, Fudan University, Shanghai 200438 China \\ ${ }^{2}$ School of Public Health, Fudan University, Shanghai 200032 China \\ ${ }^{3}$ Research Center for Ecology, College of Science, Tibet University, Lhasa 850000 China \\ ${ }^{4}$ Tibet University - Fudan University Joint Laboratory for Biodiversity and Global Change, Fudan University, Shanghai 200438 China \\ ${ }^{5}$ Fundación para el Estudio de Especies Invasivas (FuEDEI), Hurlingham, Buenos Aires 999071 Argentina \\ ${ }^{6}$ Consejo Nacional de Investigaciones Científicas y Técnicas (CONICET), Ciudad Autónoma de Buenos Aires, 999071 Argentina \\ ${ }^{7}$ Rangeland Resources \& Systems Research Unit, USDA Agricultural Research Service, Fort Collins, Colorado 80526 USA \\ ${ }^{8}$ Ecology, Department of Biology, University of Konstanz, Konstanz 78464 Germany \\ ${ }^{9}$ Zhejiang Provincial Key Laboratory of Plant Evolutionary Ecology and Conservation, Taizhou University, Taizhou 318000 China
}

Citation: Liu, M., Y. Pan, X. Pan, A. Sosa, D. M. Blumenthal, M. Van Kleunen, and B. Li. 2021. Plant invasion alters latitudinal pattern of plant-defense syndromes. Ecology 102(12):e03511. 10.1002/ecy.3511

Abstract. The relationship between herbivory and latitude may differ between native and introduced populations of invasive plants, which can generate latitudinal heterogeneity in the strength of enemy release. However, still little is known about how latitudinal heterogeneity in herbivore pressure influences latitudinal variation in defense phenotypes of invasive plants. We tested how latitudinal patterns in multi-variate defense syndromes differed between native (Argentinian) and introduced (Chinese) populations of the invasive herb Alternanthera philoxeroides. In addition, to better understand the drivers underlying latitudinal patterns, we also tested whether associations of defense syndromes with climate and herbivory differed between native and introduced ranges. We found that native plant populations clustered into three main defense syndromes associated with latitude. In contrast, we only found two defense syndromes in the introduced range. One matched the highlatitude syndrome from the native range, but was distributed at both the northern and southern range limits in the introduced range. The other was unique to the introduced range and occurred at mid-latitudes. Climatic conditions were associated with variation in syndromes in the native range, and climatic conditions and herbivory were associated with variation in syndromes in the introduced range. Together, our results demonstrate that plants may under the new environmental conditions in the introduced range show latitudinal patterns of defense syndromes that are different from those in their native range. This emphasizes that geographical dependence of population differentiation should be explicitly considered in studies on the evolution of defense in invasive plants.

Key words: alien plant; biogeography and macroecology; enemy release hypothesis; herbivore pressure; latitude; plant-defense syndromes; plant-herbivore interactions.

\section{INTRODUCTION}

Plant-herbivore interactions can vary predictably with latitude. The latitudinal herbivory-defense hypothesis (LHDH) posits that plants suffer from greater herbivore pressure and therefore evolve higher levels of defense at lower latitudes (Coley and Aide 1991, Pennings et al. 2009, Rasmann and Agrawal 2011). The generality of this hypothesis has been called into question as latitudinal trends in herbivory and plant defense are highly

Manuscript received 23 October 2020; revised 8 April 2021; accepted 17 May 2021; final version received 29 July 2021. Corresponding Editor: James T. Cronin.

${ }^{10}$ Corresponding Author. E-mail: xypan@fudan.edu.cn variable in strength and direction (Moles et al. 2011), and the alternative range center hypothesis posits a hump shaped relationship between latitude and herbivory or defense (Garcia et al. 2000, Woods et al. 2012). Nevertheless, latitudinal gradients in herbivory and defense seem to be common for native plants (Bhattarai et al. 2017, Yang et al. 2021).

Geographic patterns in plant-herbivore interactions may change during the spread of invasive plants as a result of altered herbivore communities and of having had insufficient time for evolution of gradients that parallel the ones in the native range (Bezemer et al. 2014, Cronin et al. 2015, Allen et al. 2017, Bhattarai et al. 2017, Yang et al. 2021). For example, herbivory of 
Phragmites australis in the field decreased with latitude among native populations, but not among introduced populations (Cronin et al. 2015). This non-parallel latitudinal gradients between native and introduced populations may result in greater herbivore pressure on native than introduced populations (i.e., enemy release) at low latitudes but no or less difference in herbivore pressure at higher latitudes (Bezemer et al. 2014, Cronin et al. 2015). Consequently, introduced populations may experience different evolutionary trajectories along latitude, which may confound inferences on evolutionary changes in defense phenotypes of invasive plants (Colautti et al. 2009, Yang et al. 2021). While there is increasing awareness of this issue, the incorporation of such large-scale heterogeneity in plant-herbivore interactions into studies of the evolution of invasive plants defenses remains an open and important task.

Another challenge in studying the evolution of invasive plants defenses is that plant defense phenotypes are inherently multidimensional, including many different chemical and morphological characteristics (Duffey and Stout 1996, Agrawal and Fishbein 2006). For example the general idea that invasive plants may adapt to enemy release by evolving lower defense investment (evolution of increased competitive ability or EICA; Blossey and Notzold 1995), becomes more complicated when one considers different types of defenses (Zhang et al. 2018, Liu et al. 2019). The shifting defense hypothesis (SDH) predicts an increase in toxins (e.g., flavonoids), but a decrease in digestibility reducers (e.g., tannins) and physical defenses in introduced populations due to an increase in the abundance of generalist relative to specialist enemies (Müller-Schärer et al. 2004, Zhang et al. 2018, Liu et al. 2019).

The categorization of phenotypes based on a combination of covarying traits (a so-called "syndrome" approach), is an increasingly popular framework for studying the evolution of invasive species, including the defense phenotypes (Kueffer et al. 2013, Tewes and Mueller 2018). This framework considers that the adaptation of invasive species to novel environments depends on the proper combination of multiple co-varying traits (van Kleunen et al. 2010, Kueffer et al. 2013, Leffler et al. 2014, Tewes and Mueller 2018). With regard to plant defense, Agrawal and Fishbein (2006) formalized a "defense-syndrome-triangle" hypothesis that recognizes three major defense syndromes: "low nutritional quality," "intermediate nutritional quality with chemical or physical defense," and "tolerance/escape." Applied to evolution of invasive plant defense, the question has become whether defense syndromes differ between native and introduced populations (Tewes and Mueller 2018). To our knowledge, however, the defense syndrome approach has not previously been applied to the question of whether native and introduced populations differ in latitudinal pattern of defense syndromes.

To test whether there is a latitudinal pattern in defense syndromes, and whether it differs between native and introduced populations, we characterized defense syndromes of populations of the invasive plant Alternanthera philoxeroides along latitudinal gradients in the native (Argentinian) and introduced (Chinese) ranges. In addition, as latitudinal phenotypic clines often result from underlying environmental gradients, we also tested how defense syndromes were associated with climate and herbivore pressure, and whether these associations differed between both ranges. Our common garden experiment showed that $A$. philoxeroides can simultaneously employ multiple defense traits against herbivores, including morphological traits (e.g., specific stem length), physical (e.g., trichome) and chemical defense (e.g., triterpenoid saponins; Liu et al. 2018). In addition, previous field surveys showed a shift in the composition of the herbivore community in the introduced range, as A. philoxeroides is fed on by at least 40 herbivore species in the native range (Sosa et al. 2004), but primarily fed on by a biocontrol specialist, Agasicles hygrophila (Coleoptera: Chrysomelidea) in the introduced range (data not shown). Accordingly, we asked two main questions: (1) Do populations converge into discernible defense syndromes along the latitudinal gradient, and if so, do latitudinal patterns of defense syndromes differ between native and introduced ranges? (2) Are defense syndromes associated with variation in climate or herbivory, and do these associations differ between native and introduced ranges? We expected that (1) native populations will cluster into syndromes of the defensesyndrome triangle (Agrawal and Fishbein 2006), and the syndromes with higher overall levels of defense will be more prevalent in the native range (following EICA and $\mathrm{SDH}$ ), particularly at low latitudes (following from the LHDH). In contrast, introduced populations may show a weaker latitudinal pattern of defense syndromes than native populations as a consequence of altered herbivore communities and insufficient time to evolve. We also expected that (2) defense syndromes will be associated with climate and herbivory, and these associations will be weaker in the introduced than in the native range.

\section{Methods \\ Study species}

Alternanthera philoxeroides is an herbaceous plant of wetlands and adjacent terrestrial habitats, native to South America and invasive in parts of Asia, Oceania, and North America (Pan et al. 2007). In China, $A$. philoxeroides was introduced in the 1930s and has become one of the most noxious weeds in wetlands and agroecosystems (Pan et al. 2007). Recent investigations showed that it has widely dispersed in southern China and expanded its distribution up to a latitude of $38^{\circ} \mathrm{N}$ (Lu et al. 2013). Alternanthera philoxeroides is known to be fed on by at least 40 herbivore species in its home range, including at least five specialists (Sosa et al. 2004). In China, Agasicles hygrophila is the primary 
specialist insect herbivore on $A$. philoxeroides, and was first introduced in 1987 as a biological control agent (Ma 2001).

\section{Data collection}

To assess variation in herbivory and defense, we conducted field surveys in terrestrial habitats of $A$. philoxeroides. One was in 17 populations along a latitudinal transect from $25.2^{\circ} \mathrm{S}$ to $37.8^{\circ} \mathrm{S}$ in the native range, Argentina, and the other was in 24 populations along a latitudinal transect from $21.9^{\circ} \mathrm{N}$ to $36.8^{\circ} \mathrm{N}$ in the introduced range, China (Appendix S1: Table S1). These transects covered the major latitudinal distribution of A. philoxeroides in each range. In each population, we selected four quadrats $(1 \times 1 \mathrm{~m}, \sim 10 \mathrm{~m}$ apart $)$ along a $30-\mathrm{m}$ transect and randomly collected 30 plants per quadrat to measure (1) plant morphology (specific leaf area [SLA], specific stem length [SSL]), (2) a putative physical defense (trichome density), (3) putative chemical defenses (triterpenoid saponins, flavonoids, tannins, lignin), (4) nutritional value (carbon : nitrogen ratio [C:N ratio]), and (5) leaf herbivory. The quantification methods are provided in Appendix S1: Section S1.

We chose those traits because they represent plant functional dimensions along three axes: plant growth (SLA, C:N ratio), resistance against abiotic stress (SLA), and defenses against herbivores (trichome density, secondary metabolites, C:N ratio; Karban and Baldwin 1997, Wright et al. 2004, Cornelissen et al. 2008, Diaz et al. 2016). The secondary metabolites can be subdivided into toxins (triterpenoid saponins, flavonoids) and digestibility-reducing compounds (tannins, lignin). Specific stem length is a combination of stem thickness and density; stems with a higher SSL are typically thin and have higher structural defenses against stem-boring herbivores in A. philoxeroides (Pan et al. 2011).

To characterize the climatic conditions at each site (population), we extracted eight climatic variables from the BIOCLIM database (Hijmans et al. 2005; database available online). ${ }^{11}$ Specifically, we used BIO1 (mean annual temperature, ${ }^{\circ} \mathrm{C}$ ), BIO4 (temperature seasonality, expressed as the standard deviation of temperature among months $\times 100$ ), BIO5 (maximum temperature of the warmest month, ${ }^{\circ} \mathrm{C}$ ), BIO6 (minimum temperature of the coldest month, ${ }^{\circ} \mathrm{C}$ ), BIO12 (mean annual precipitation, $\mathrm{mm}$ ), BIO13 (precipitation of the wettest month, $\mathrm{mm}$ ), BIO14 (precipitation of the driest month, $\mathrm{mm}$ ), and BIO15 (precipitation seasonality, expressed as standard deviation of precipitation across months) as climatic variables. These variables denote annual means of climate, climatic extremes, and seasonality (Appendix S1: Table S2; Fig. S1). We summarized climatic variables of the native and introduced ranges by

\footnotetext{
${ }^{11} \mathrm{http}: / /$ www.worldclim.org/
}

conducting principal component analyses (PCA). The first principal component axis, climate $\mathrm{PC} 1$, explained $50.4 \%$ of the variation and primarily showed negative associations with mean annual temperature, mean annual precipitation and minimum temperature of the coldest month. The second axis, climate PC2, explained $39.6 \%$ of the variation and mainly showed a negative relationship with precipitation of the driest month, and positive relationships with maximum temperature of the warmest month and precipitation seasonality (Appendix S1: Table S2).

\section{Statistical analysis}

Grouping of populations into syndromes. - To detect potential defense syndromes, we averaged the data of the four quadrats within each population and performed hierarchical clustering using the populations as units. Clustering was done with the hclust command in the $\mathrm{R}$ package stats based on Ward's linkage method and Euclidean distances. This clustering method combines populations to find subgroups that minimize the withincluster ANOVA sum-of-squares at each iteration (Murtagh and Legendre 2014). Because the hierarchical clustering needs to specify the number of clusters, we set the optimal number of clusters to four based on the gap statistic (function clusGap in the $\mathrm{R}$ package cluster; Maechler et al. 2019; see Appendix S1: Fig. S2 for gap statistic results).

To show how each defense syndrome is ordinated in two-dimensional space, we then performed a PCA with the rda command of the vegan $\mathrm{R}$ package, with default settings (Oksanen et al. 2019). The PCA was also performed based on the $8 \times 41$ traits-by-population matrix, in which data were transformed to $Z$ scores (mean $=0, \mathrm{SD}=1)$, so that they were measured on a comparable scale. The first two principal components (i.e., defense PC1 and defense PC2) were extracted for statistically assessing the variation of the plant-defense syndromes along the latitudinal, climatic and herbivory gradients.

To visualize the differences between defense syndromes, we characterized each defense syndrome using radial plots (Defossez et al. 2018, Formenti et al. 2021). These were built by plotting the scaled values of each trait as the distances from the center of a circular field along each direction (one direction per trait). The trait values plotted are the means of each trait averaged over all populations assigned to that syndrome, and the position of the axis was defined by the order of the variables in the PCA.

Statistical analyses along the gradients. - To test whether there were latitudinal pattern of defense syndromes and whether these differed between the native and introduced ranges, we performed permutational multivariate analysis of variance (PERMANOVA). We used either the raw $8 \times 41$ traits-by-population matrix or the first 
two principal components of defense traits as dependent variables, and included latitude, range and latitude $\times$ range as explanatory variables. Random permutations were performed 9999 times. The PERMANOVA tests were conducted using the anova.cca command in vegan package (Oksanen et al. 2019).

To assess whether the latitudinal patterns in defense syndromes reflect associations with climate and/or herbivory pressure, we also ran PERMANOVAs in which we replaced latitude with the first and second principal components of climate variables (CPC1 and CPC2) and herbivory. These models also included three-way and four-way interactions between the explanatory variables. If there was a significant interaction of range with one of the environmental factors (climate or herbivory), we visualized this result with three-dimensional plots of defense PC1, defense PC2 and the environmental factor for the native and introduced ranges, respectively.

Additionally, to gain insight into which defense traits drive patterns in the defense syndromes among populations, we also did univariate analyses for herbivory and each of the measured plant defensive traits. For these univariate tests, we used a generalized linear mixed model (GLMM) with a log link function and Gaussian error distribution for leaf herbivory and linear mixed models (LMMs) for each of the eight defensive traits. These models included range, latitude, latitude ${ }^{2}$, and the interactions between range and latitude, and between range and latitude ${ }^{2}$ as fixed effects. The unit of analysis in these models was the average trait value per quadrat (i.e., four values per population). Therefore, population was treated as a random effect. We provided a more detailed description of these univariate tests in Appendix S1: Section S1, and we present the results in Appendix S1: Fig. S3, Tables S3, S4. The least-square mean of herbivory and each defensive trait in each range were calculated using the emmeans command in the emmeans package (Lenth 2020), and the results are reported in Appendix S1: Table S5.

\section{RESULTS}

\section{Plant-defense syndromes along latitudinal gradients in the native and introduced ranges}

The four clusters extracted from the hierarchical clustering analysis explained $54.8 \%$ of the total variability in plant defensive traits (Fig. 1a). These clusters represented four defense syndromes, which significantly separated along latitude (significant latitude effect in Appendix S1: Table S6a), and the latitudinal patterns differed significantly between the native and introduced ranges (range $\times$ latitude interaction in Appendix S1: Table S6a).

Syndrome $1_{\text {native }}$ (low nutritional quality/high abiotic resistance strategy, orange area in Fig. 1) included populations with high levels of digestibility reducing compounds (lignin, tannins), a low nutritional quality (high
$\mathrm{C}: \mathrm{N}$ ratios) and a high abiotic resistance (low SLA) (Fig. 1b), and was present at low latitudes in the native range (Fig. 1c).

Syndrome $2_{\text {native }}$ (intermediate nutritional quality and high chemical defense strategy, green area in Fig. 1) included populations with intermediate levels of nutritional quality (intermediate $\mathrm{C}: \mathrm{N}$ ratios), low levels of digestibility reducing compounds (low tannins), and high levels of toxins (high triterpenoid saponins and flavonoids) (Fig. 1b), and was present at intermediate latitudes in the native range (Fig. 1c).

Syndrome 3 native \& introduced (fast growth/tolerance/ escape strategy, blue area in Fig. 1) included populations with fast growth (high SLA), high levels of nutritional quality (low C:N ratio), and low levels of physical and chemical defense (low trichome density, triterpenoid saponins and tannins) (Fig. 1b), and was present at high latitudes in the native range, and at high and low latitudes in the introduced range (Fig. 1c).

Syndrome $2_{\text {introduced }}$ (intermediate nutritional quality and high physical defense strategy, yellow in Fig. 1) included populations with intermediate level of nutritional quality (intermediate $\mathrm{C}: \mathrm{N}$ ratio), but high levels of physical defense (trichome density) and SSL (Fig. 1b), and was present at intermediate latitudes in the introduced range (Fig. 1c).

\section{Relationships of the defense syndromes with climate and herbivory}

We found that the plant-defenses distance matrix, irrespective of whether it was based on all of the defensive traits or on the first two principal components of plant defense (defense PC1 and PC2), was significantly related to climatic conditions as well as herbivory levels (significant CPC1, CPC2, and herbivory effects, Appendix S1: Table S6b). Moreover, these relationships differed between the native and introduced ranges (significant interactions of range with $\mathrm{CPC} 1, \mathrm{CPC} 2$, and herbivory, Appendix S1: Table S6b). In the native range, the three syndromes characterized by defense PC1 and defense PC2 showed significant correlations with climate PC1 $\left(F_{3,13}=4.37 ; P=0.025 ; R^{2}=0.425\right)$ and climate $\mathrm{PC} 2$ values $\left(F_{3,13}=52.22 ; P<0.001 ; R^{2}=0.414\right)$, but were not correlated with herbivory levels $\left(F_{3,13}=2.210\right.$; $P=0.135 ; R^{2}=0.061$, Fig. $\left.2 \mathrm{a}, \mathrm{c}, \mathrm{e}\right)$. In the introduced range, the two syndromes showed significant correlations with climate PC2 $\left(F_{3,21}=62.91 ; P<0.001 ; R^{2}=\right.$ $0.313)$ and herbivory levels $\left(F_{3,21}=69.316 ; P<0.001\right.$; $\left.R^{2}=0.464\right)$, but were not correlated with climate PC1 $\left(F_{3,21}=0.254 ; P=0.858 ; R^{2}=0.057\right.$, Fig. $\left.2 \mathrm{~b}, \mathrm{~d}, \mathrm{f}\right)$.

\section{Discussion}

Our results showed that (1) native and introduced populations of $A$. philoxeroides differed in the latitudinal pattern of defense syndromes. Specifically, native populations were clustered into three main defense 
a
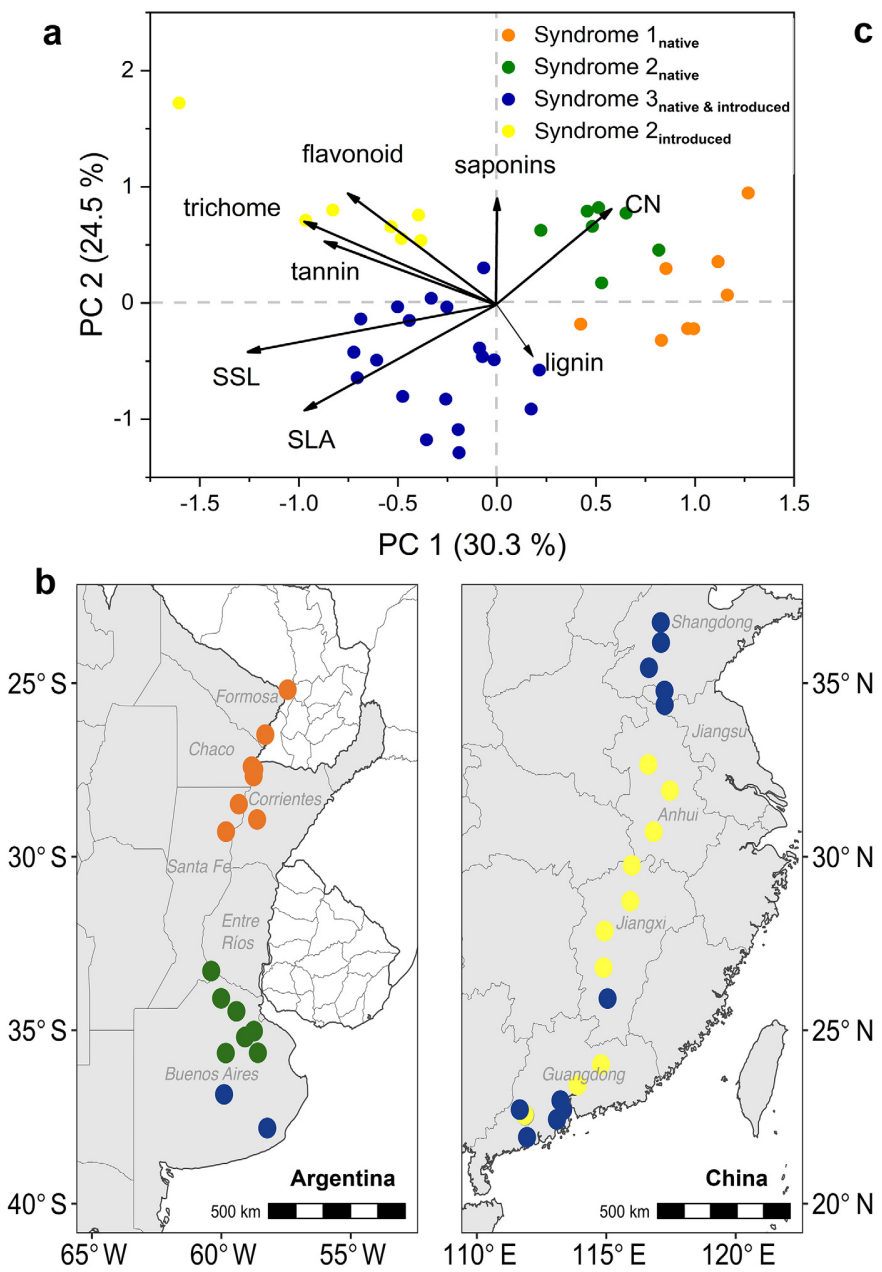

C
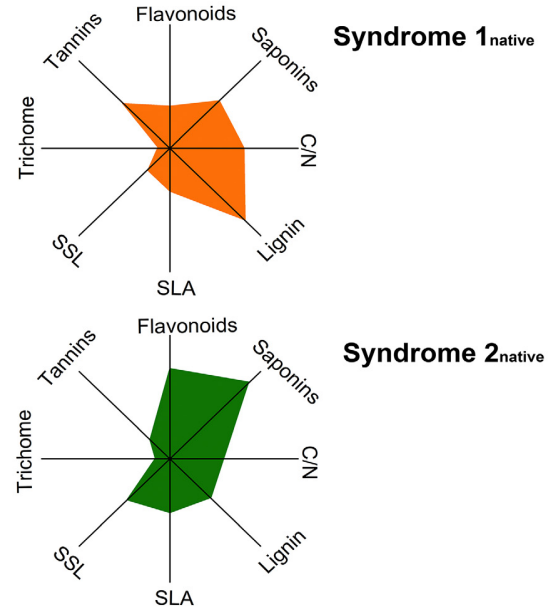

Syndrome 2native

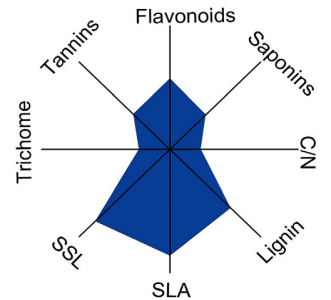

Syndrome 3 native \& introduced

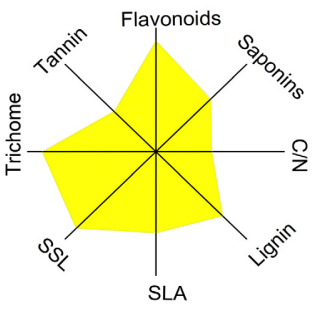

Syndrome 4 introduced

FIg. 1. Ordination, radial plots, and latitudinal distribution of the four plant-defense syndromes. (a) Scatter plot of principal component analysis of defense trait variation: each dot represents one of the 41 sampled populations, and only the first two PCA axes are shown. (b) Radial plot of the four defense syndromes, in which each axis of the radial plot corresponds to one of the eight defense-related traits. The order of the traits corresponds to the direction of the trait vector in the PCA of panel a and trait values represent the mean of traits over all populations assigned to that syndrome. (c) Latitudinal distribution of defense syndromes in the native (Argentinian) and introduced (Chinese) range. Each dot represents one of the sampled populations, and their colors match those of the dominant plant-defense syndrome across the four quadrats sampled within each population.

syndromes, which were found at different latitudes. In contrast, introduced populations were clustered into only two defense syndromes (Fig. 1). One was the same syndrome that was present at high latitudes in the native range, but with a disjunct distribution at high and low latitudes in the introduced range. The other syndrome was unique to intermediate latitudes of the introduced range. (2) Defense syndromes were associated with climate but not herbivory in the native range. In contrast syndromes related to both climate and herbivory in the introduced range. Specifically, well defended phenotypes were present at mid latitudes, which had higher herbivore pressure (Fig. 2), likely due to an abundant biological control agent. Together, these findings suggest that the novel climates and herbivore communities encountered by invasive plants, driven by biological control as well as enemy release, can together lead to distinct latitudinal patterns of defense.

We identified four defense syndromes in A. philoxeroides based on trait covariance (Fig. 1a), and found that these defense syndromes were non-randomly distributed across latitudes, both in native and introduced range. In the native range, defense syndromes shifted from a low nutritional quality/high abiotic resistance strategy (syndrome $1_{\text {native}}$ ) at low latitudes via an intermediate nutritional quality and high chemical defense strategy (syndrome 2 native) at intermediate latitudes to a fast growth/tolerance/escape strategy (syndrome 3 native \& introduced) at high latitudes. These patterns suggest that overall levels of defense fit with the latitudinal herbivory-defense hypothesis (Coley and Aide 1991, Pennings et al. 2009, Rasmann and Agrawal 2011), 
Native range
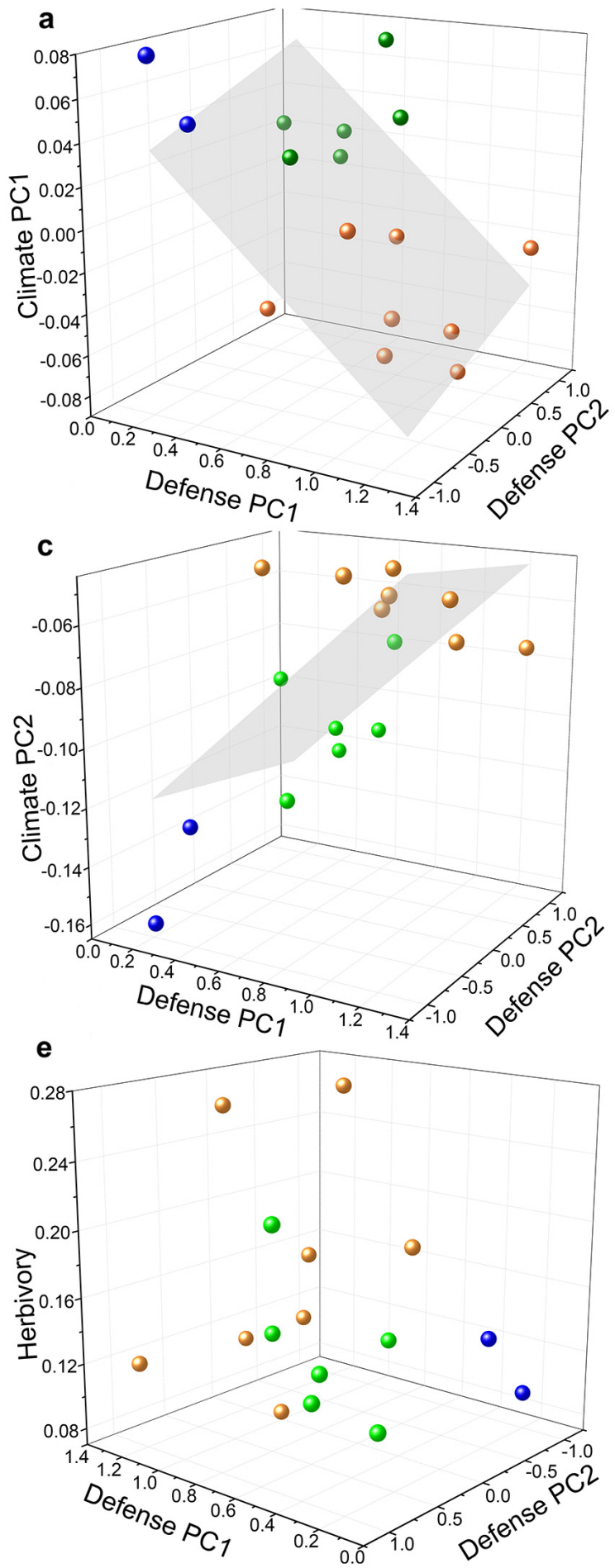

Introduced range
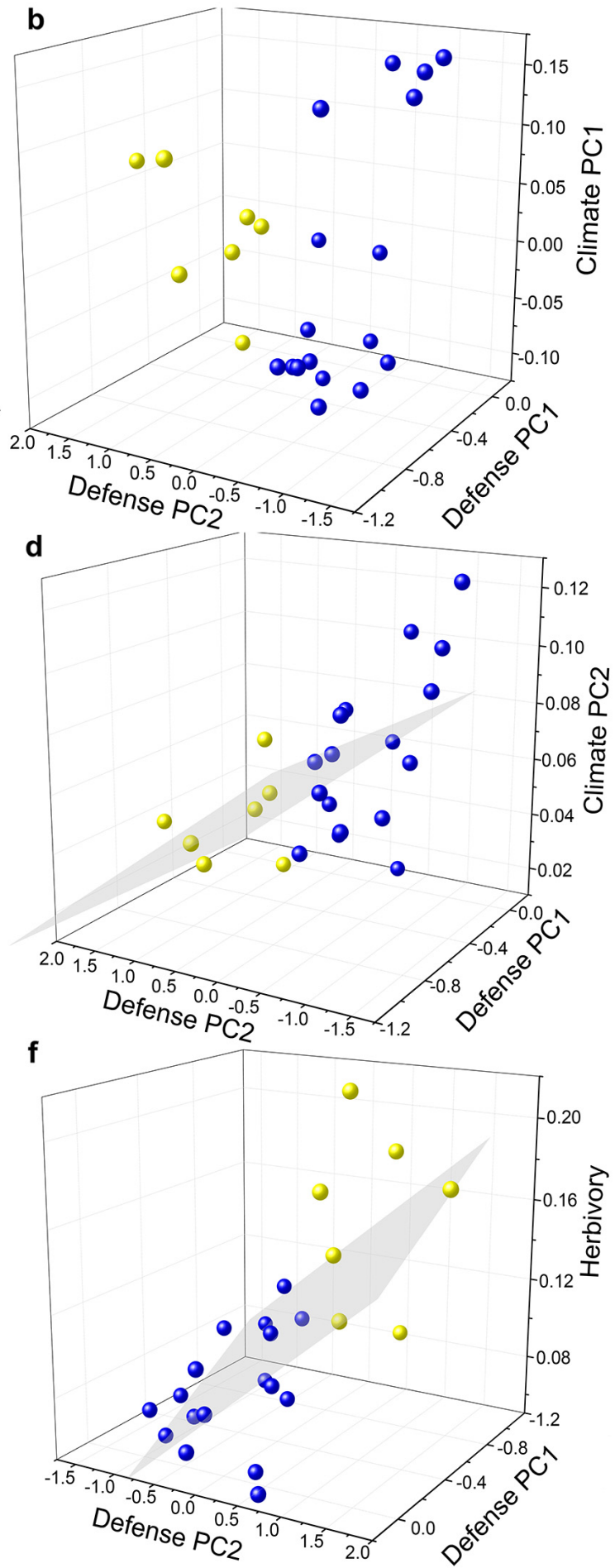

FIG. 2. Relationships of the defense syndromes (represented by defense PC1 and defense PC2) with climate and herbivory. (a-d) Correlations of defense PC1 and PC2 with climatic conditions, represented by the first and second axis of the climatic PCA (Climate PC1 captured the variation in the annual means of temperature and precipitation, and climate PC2 mainly captured variation in extremes and seasonality of temperature and precipitation in Appendix S1: Table S2). (e, f) Correlations of defense PC1 and PC2 with the average herbivory damage measured for each population. The grey shading represents the regression plane. Dots represent population averages, and colors represent the four syndromes as detailed in Fig. 1. 
despite mixed results for individual defensive traits (Appendix S1: Fig. S3). Our finding of three defense syndromes in the native range of $A$. philoxeroides is also consistent with a defense syndrome triangle hypothesis (Agrawal and Fishbein 2006), and our previous observation of three morphologically distinct varieties of $A$. philoxeroides at different latitudes in the native range (Jia et al. 2010). The distinct pattern of defense syndromes in the introduced range, where the better defended populations are found at mid latitudes, is in line with the predictions of the range center hypothesis (Garcia et al. 2000, Woods et al. 2012). However, it may also be driven by the current range of the biological control agent $A$. hygrophilla in China. Thus, our findings provide empirical evidence for a difference in geographical patterns of defense syndromes between native and introduced populations.

Our results provide insight into the potentially complex eco-evolutionary scenarios underlying invasion success of $A$. philoxeroides. The establishment of syndrome $3_{\text {native } \& \text { introduced }}$ (fast growth/tolerance/escape strategy) populations within the southern and northern limits of the introduced range suggests adaptation to local extreme climatic conditions and lower leaf damage by the biocontrol agent $A$. hygrophila (Figs. 2 and 3 h). Similar associations are likely present in the northern part of the native range, which would suggest that

a) Native range

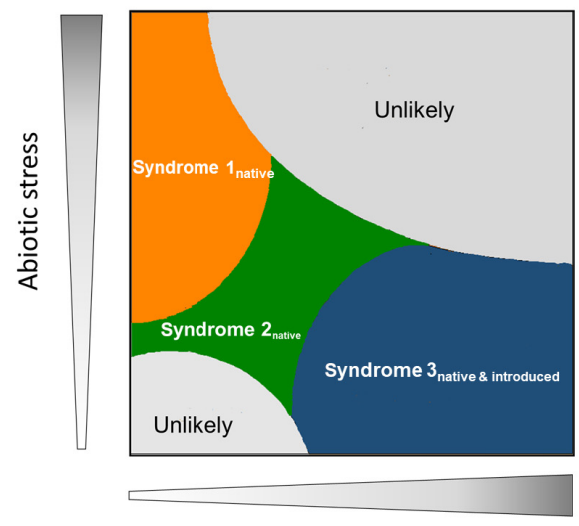

Herbivore pressure preadaptation may be important for A. philoxeroides invasion at the margins of its climatic range (Yang et al. 2021). The establishment of syndrome $2_{\text {introduced }}$ (high nutritional value and high physical defense strategy) populations at intermediate latitudes of the introduced range suggests adaptation to mild climatic conditions and high herbivory pressures by A. hygrophila (Figs. 2 and $3 \mathrm{~h}$ ). We speculate that this syndrome could have arisen from the introduction of native syndrome $1_{\text {native, }}$ followed by adaptation to the fertile, frequently eutrophied, environment of central China (Xu et al. 2020), or a shift in herbivore composition from both specialists and generalists at intermediate latitudes in the native range to an abundance of the specialist $A$. hygrophila at intermediate latitudes the introduced range (MüllerSchärer et al. 2004, Sosa et al. 2004, Lu et al. 2013). Hence, our results suggest that the introduced populations of $A$. philoxeroides may experience different evolutionary trajectories across latitudes.

Our results further showed that the biogeographical heterogeneity in the strength of enemy release may confound the inference of evolutionary changes of plant defense in invasive plants. We found that leaf herbivory in the native range linearly decreased with latitude, whereas the relationship was hump-shaped for the introduced range (Appendix S1: Fig. S3). The net effect is that A. philoxeroides in China still experiences enemy release

\section{b) Introduced range}

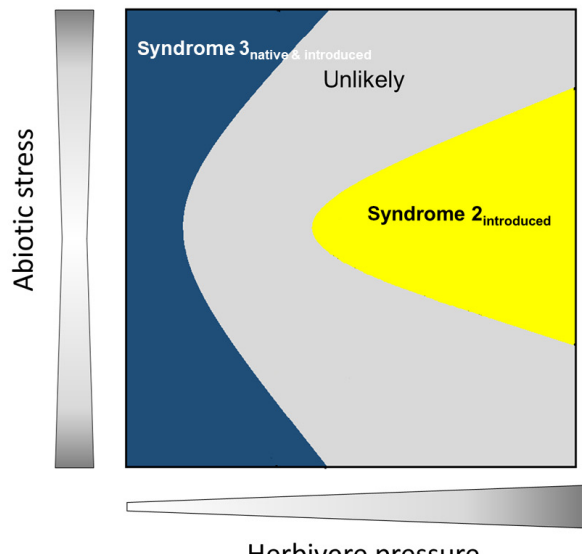

Herbivore pressure

FIG. 3. A conceptual framework for the positions of the defense syndromes along ecological gradients in the native and introduced range. (a) The positions of the three syndromes along ecological gradients in native range. The low nutritional quality/high abiotic resistance strategy (syndrome $1_{\text {native }}$, orange) is found in environments with high abiotic stress. The intermediate nutritional and high chemical defense strategy (syndrome $2_{\text {native, }}$ green) is found in environments with moderate levels of abiotic stress and herbivory environment. The fast growth/tolerance/escape strategy (syndrome $3_{\text {native }} \&$ introduced, blue) is found in environments with low abiotic stress and high levels of herbivory. (b) The positions of the two syndromes along ecological gradients in the introduced range. Syndrome 3 native \& introduced (blue; the same syndrome as in the native range) is here found in environments with low or high abiotic stress and low levels of herbivory environment, and is the same syndrome in the high latitude of native range. The intermediate nutritional quality/high physical defense strategy (syndrome $2_{\text {introduced, }}$ yellow) is found in environments with low abiotic stress and high levels of herbivory, and is unique to the introduced range. The gray areas represent zones of empty environmental space, where no populations of Alternanthera philoxeroides were found in this study. 
at high and low latitudes, but not at intermediate latitudes. This finding supports previous work showing latitudinal heterogeneity in the strength of enemy release (Cronin et al. 2015, Allen et al. 2017, Bhattarai et al. 2017, Allen et al. 2020). As an evolutionary extension of the enemy release hypothesis, the evolution of increased competitive hypothesis (EICA), which predicts that introduced plants should evolve increased growth and reproduction, and reduced defense (Blossey and Notzold 1995), has stimulated studies on the role of rapid adaptive evolution in the establishment and spread of invasive species (Huey et al. 2000). Like many other studies (Bossdorf et al. 2005, Felker-Quinn et al. 2013, Zhang et al. 2018), comparisons of the mean values of the defense traits between the introduced and native range provided partial evidence for reduced defense in $A$. philoxeroides' introduced range (Appendix S1: Table S5). One possible explanation for such inconsistent results could be that latitudinal variation may confound comparisons between ranges (van Kleunen and Fischer 2008, Colautti et al. 2009). This confounding effect became apparent when comparing defense syndromes. In the invasion of $A$. philoxeroides, it appears that enemy release does coincide with reductions in defense, but these effects are geographically restricted to northern and southern latitudes, likely due to the presence of a biological control herbivore at intermediate latitudes ( $\mathrm{Lu}$ et al. 2013). Therefore, our findings demonstrate that the difference between native and introduced populations is dependent on environmental factors that vary with latitude. This emphasizes that such latitudinal dependence of population differentiation should be explicitly considered in the experimental design and statistical analysis of studies of the evolution of defense in invasive plants (Bhattarai et al. 2017, Allen et al. 2020, Croy et al. 2020).

Our results showed that associations between defense syndromes and environmental factors were partly different between the native and introduced range of $A$. philoxeroides. For example, defense syndromes were significantly associated with annual mean temperature and precipitation (climate $\mathrm{PC} 1$ ) in the native range, but not in the introduced range. The significant association with climate $\mathrm{PC} 1$ in the native range is in accord with previous studies showing that climate can affect defense phenotypes by imposing physiological constraints and determining the availability of resources to plants (Loughnan and Williams 2019). However, plant-defense syndromes were not associated with leaf herbivory in the native range. One possible explanation is that our leafdamage measurements may not represent overall herbivore pressure in the native range (Salazar and Marquis 2012, Anstett et al. 2016). For example, we likely underestimated damage from the guild of leaf-suckers like a specialist herbivore, Amynothrips andersoni, and large vertebrate grazers (e.g., cattle), which were abundant in high-latitude Pampas grassland of the native range (M. Liu, personal observation), but absent from the introduced range.
By contrast, defense syndromes were related to both herbivory and climate (climate PC2) in the introduce range. The herbivory pressure in the introduced range is primarily imposed by $A$. hygrophila, an obligate specialist introduced to China as a biological control agent in 1987 (Ma 2001). A. hygrophila is sensitive to extreme climatic conditions at both high and low latitudes (Zhao et al. 2015, Jia et al. 2020), and consequently is most abundant at intermediate latitudes ( $\mathrm{Lu}$ et al. 2013). The climate of the introduced range is more extreme and variable (e.g., lower precipitation of driest month and higher precipitation seasonality, Appendix S1: Fig. S1) at high and low latitudes than that at intermediate latitudes. Therefore, our results suggest that the plant-defense syndromes of introduced populations are driven by the abundance of $A$. hygrophila, which in turn is driven by climatic conditions that vary with latitude in the introduced range.

While the variation in defense traits that we found might be shaped by different selection pressures, it is important to bear in mind that our field survey does not allow us to determine whether genetic differences underlie the observed geographic variation in defense traits. Common garden comparisons would be needed to assess the roles of phenotypic plasticity and genetic differentiation in driving this biogeographical variation in plant defense (Yang et al. 2021). Nevertheless, our study on defense syndromes along latitudes and between the native and introduced range of $A$. philoxeroides contributes to a better understanding of the geography of plant defense.

\section{ACKNOWLEDGMENTS}

The work was supported by the National Natural Science Foundation of China (Grant No. 41771053; No. 32071659; No. 31961133028; No. 32030067). We thank Zhijie Zhang and Yang Yang for advice on the writing and are grateful to Liangrui $Y u$, Yaolin Guo, Kehao Xu, and other members of IBSFU for help in the research. Mu Liu and Xiaoyun Pan developed manuscript ideas, Mu Liu, Yuanfei Pan, and Alejandro Sosa carried out data collection. Mu Liu and Yuanfei Pan analyzed the data and wrote the manuscript with advice from Xiaoyun Pan, Dana M. Blumenthal, Mark van Kleunen, and Bo Li. Mu Liu and Yuanfei Pan should be considered joint first authors.

\section{Literature Cited}

Agrawal, A. A., and M. Fishbein. 2006. Plant defense syndromes. Ecology 87:132-149.

Allen, W. J., A. E. DeVries, N. J. Bologna, W. A. Bickford, K. P. Kowalski, L. A. Meyerson, and J. T. Cronin. 2020. Intraspecific and biogeographical variation in foliar fungal communities and pathogen damage of native and invasive Phragmites australis. Global Ecology and Biogeography 29:1199-1211.

Allen, W. J., L. A. Meyerson, D. Cummings, J. Anderson, G. P. Bhattarai, and J. T. Cronin. 2017. Biogeography of a plant invasion: drivers of latitudinal variation in enemy release. Global Ecology and Biogeography 26:435-446.

Anstett, D. N., K. A. Nunes, C. Baskett, and P. M. Kotanen. 2016. Sources of controversy surrounding latitudinal patterns in herbivory and defense. Trends in Ecology \& Evolution 31:789-802. 
Bezemer, T. M., J. A. Harvey, and J. T. Cronin. 2014. Response of native insect communities to invasive plants. Annual Review of Entomology 59:119-141.

Bhattarai, G. P., L. A. Meyerson, J. Anderson, D. Cummings, W. J. Allen, and J. T. Cronin. 2017. Biogeography of a plant invasion: genetic variation and plasticity in latitudinal clines for traits related to herbivory. Ecological Monographs 87:57-75.

Blossey, B., and R. Notzold. 1995. Evolution of increased competitive ability in invasive nonindigenous plants - a hypothesis. Journal of Ecology 83:887-889.

Bossdorf, O., H. Auge, L. Lafuma, W. E. Rogers, E. Siemann, and D. Prati. 2005. Phenotypic and genetic differentiation between native and introduced plant populations. Oecologia 144:1-11.

Colautti, R. I., J. L. Maron, and S. C. H. Barrett. 2009. Common garden comparisons of native and introduced plant populations: latitudinal clines can obscure evolutionary inferences. Evolutionary Applications 2:187-199.

Coley, P. D., and T. M. Aide. 1991. Comparison of herbivory and plant defenses in temperate and tropical broad-leaved forests. Pages 25-49 in P. W. Price, editor. Plant-animal interaction: evolutionary ecology in tropical and temperate regions. Wiley, New York, New York, USA

Cornelissen, T., G. W. Fernandes, and J. Vasconcellos-Neto. 2008. Size does matter: variation in herbivory between and within plants and the plant vigor hypothesis. Oikos 117:1121-1130.

Cronin, J. T., G. P. Bhattarai, W. J. Allen, and L. A. Meyerson. 2015. Biogeography of a plant invasion: plant-herbivore interactions. Ecology 96:1115-1127.

Croy, J. R., L. A. Meyerson, W. J. Allen, G. P. Bhattarai, and J. T. Cronin. 2020. Lineage and latitudinal variation in Phragmites australis tolerance to herbivory: implications for invasion success. Oikos 129:1341-1357.

Defossez, E., L. Pellissier, and S. Rasmann. 2018. The unfolding of plant growth form-defence syndromes along elevation gradients. Ecology Letters 21:609-618.

Diaz, S., et al. 2016. The global spectrum of plant form and function. Nature 529:167-173.

Duffey, S. S., and M. J. Stout. 1996. Antinutritive and toxic components of plant defense against insects. Archives of Insect Biochemistry and Physiology 32:3-37.

Felker-Quinn, E., J. A. Schweitzer, and J. K. Bailey. 2013. Metaanalysis reveals evolution in invasive plant species but little support for Evolution of Increased Competitive Ability (EICA). Ecology and Evolution 3:739-751.

Formenti, L., V. Caggia, J. Puissant, T. Goodall, G. Glauser, R. Griffiths, and S. Rasmann. 2021. The effect of root-associated microbes on plant growth and chemical defence traits across two contrasted elevations. Journal of Ecology 109:38-50.

Garcia, D., R. Zamora, J. M. Gomez, P. Jordano, and J. A. Hodar. 2000. Geographical variation in seed production, predation and abortion in Juniperus communis throughout its range in Europe. Journal of Ecology 88:436-446.

Hijmans, R. J., S. E. Cameron, J. L. Parra, P. G. Jones, and A. Jarvis. 2005. Very high resolution interpolated climate surfaces for global land areas. International Journal of Climatology 25:1965-1978.

Huey, R. B., G. W. Gilchrist, M. L. Carlson, D. Berrigan, and L. Serra. 2000. Rapid evolution of a geographic cline in size in an introduced fly. Science 287:308-309.

Jia, D., X. Yuan, Y. Liu, C. Xu, Y. Wang, L. Gao, and R. Ma. 2020. Heat sensitivity of eggs attributes to the reduction in Agasicles hygrophila population. Insect Science 27:159-169.

Jia, X., X. Pan, A. Sosa, B. Li, and J. Chen. 2010. Differentiation in growth and biomass allocation among three native Alternanthera philoxeroides varieties from Argentina. Plant Species Biology 25:85-92.
Karban, R., and I. T. Baldwin. 1997. Induced responses to herbivory. University of Chicago Press, Chicago, Illinois, USA.

Kueffer, C., P. Pysek, and D. M. Richardson. 2013. Integrative invasion science: model systems, multi-site studies, focused meta-analysis and invasion syndromes. New Phytologist 200:615-633.

Leffler, A. J., J. J. James, T. A. Monaco, and R. L. Sheley. 2014. A new perspective on trait differences between native and invasive exotic plants. Ecology 95:298-305.

Lenth, R. V. 2020. emmeans: Estimated Marginal Means, aka Least-Squares Means. R package version 1.5.3. https:// CRAN.R-project.org/package $=$ emmeans

Liu, M., et al. 2021. data.csv. Figshare, data set. https://doi.org/ 10.6084/m9.figshare.14635374.v1

Liu, M., X. Pan, Z. Zhang, M. van Kleunen, and B. Li. 2019. Testing the shifting defense hypothesis for constitutive and induced resistance and tolerance. Journal of Pest Science 93:355-364.

Liu, M., F. Zhou, X. Y. Pan, Z. J. Zhang, M. B. Traw, and B. Li. 2018. Specificity of herbivore-induced responses in an invasive species, Alternanthera philoxeroides (alligator weed). Ecology and Evolution 8:59-70.

Loughnan, D., and J. L. Williams. 2019. Climate and leaf traits, not latitude, explain variation in plant-herbivore interactions across a species' range. Journal of Ecology 107:913-922.

Lu, X., E. Siemann, X. Shao, H. Wei, and J. Ding. 2013. Climate warming affects biological invasions by shifting interactions of plants and herbivores. Global Change Biology 19:2339-2347.

Ma, R. 2001. Ecological adaptation for the intruduced biocontrol agent, Agasicles hygropghila, for alligatorweed, Alternanthera philoxeroides, in China. Chinese Academy of Agricultural Sciences, Beijing, China.

Maechler, M., et al. 2019. "Finding Groups in Data": Cluster Analysis Extended Rousseeuw et al. R package version 2.1.0. https://CRAN.R-project.org/package=cluster

Moles, A. T., S. P. Bonser, A. G. B. Poore, I. R. Wallis, and W. J. Foley. 2011. Assessing the evidence for latitudinal gradients in plant defence and herbivory. Functional Ecology 25:380 388.

Müller-Schärer, H., U. Schaffner, and T. Steinger. 2004. Evolution in invasive plants: implications for biological control. Trends in Ecology \& Evolution 19:417-422.

Murtagh, F., and P. Legendre. 2014. Ward's hierarchical agglomerative clustering method: Which algorithms implement Ward's criterion? Journal of Classification 31:274-295.

Oksanen, J., et al. 2019. vegan: Community ecology package. $\mathrm{R}$ package version 2.5-4. https://CRAN.R-project.org/package= vegan

Pan, X., Y. Geng, A. Sosa, W. Zhang, B. Li, and J. Chen. 2007. Invasive Alternanthera philoxeroides: biology, ecology and management. Acta Phytotaxonomica Sinica 45:884 900.

Pan, X., X. Jia, J. Zeng, A. Sosa, B. Li, and J. Chen. 2011. Stem tissue mass density is linked to growth and resistance to a stem-boring insect in Alternanthera philoxeroides. Plant Species Biology 26:58-63.

Pennings, S. C., C. K. Ho, C. S. Salgado, K. Wieski, N. Dave, A. E. Kunza, and E. L. Wason. 2009. Latitudinal variation in herbivore pressure in Atlantic Coast salt marshes. Ecology 90:183-195.

Rasmann, S., and A. Agrawal. 2011. Latitudinal patterns in plant defense: evolution of cardenolides, their toxicity and induction following herbivory. Ecology Letters 14:476-483.

Salazar, D., and R. J. Marquis. 2012. Herbivore pressure increases toward the equator. Proceedings of the National Academy of Sciences USA 109:12616-12620. 
Sosa, A., M. H. Julien, and H. Cordo. 2004. New research on Alternanthera philoxeroides (alligator weed) in its South American native range. Pages 180-185 in J. M. Cullen, D. T. Briese, D. J. Kriticos, W. M. Lonsdale, J. M. Cullen, D. T. Briese, D. J. Kriticos, and W. M. Lonsdale, editors. Proceedings of the XI international symposium on biological control of weeds. CSIRO Entomology, Canberra, Australia.

Tewes, L. J., and C. Mueller. 2018. Syndromes in suites of correlated traits suggest multiple mechanisms facilitating invasion in a plant range-expander. Neobiota 37:1-22.

van Kleunen, M., and M. Fischer. 2008. Adaptive rather than non-adaptive evolution of Mimulus guttatus in its invasive range. Basic and Applied Ecology 9:213-223.

van Kleunen, M., E. Weber, and M. Fischer. 2010. A metaanalysis of trait differences between invasive and non-invasive plant species. Ecology Letters 13:235-245.

Woods, E. C., A. P. Hastings, N. E. Turley, S. B. Heard, and A A. Agrawal. 2012. Adaptive geographical clines in the growth and defense of a native plant. Ecological Monographs $82: 149-168$

Wright, I. J., et al. 2004. The worldwide leaf economics spectrum. Nature 428:821-827.

Xu, X., H. Liu, Y. Liu, C. Zhou, L. Pan, C. Fang, M. Nie, and B. o. Li. 2020. Human eutrophication drives biogeographic salt marsh productivity patterns in China. Ecological Applications 30:e2045.

Yang, Y., et al. 2021. Rapid evolution of latitudinal clines in growth and defense of an invasive weed. New Phytologist 230:845-856

Zhang, Z., X. Pan, D. Blumenthal, M. van Kleunen, M. Liu, and B. Li. 2018. Contrasting effects of specialist and generalist herbivores on resistance evolution in invasive plants. Ecology 99:866-875

Zhao, L., D. Jia, X. Yuan, Y. Guo, W. Zhou, and R. Ma. 2015. Cold hardiness of the biological control agent, Agasicles hygrophila, and implications for its potential distribution. Biological Control 87:1-5.

\section{SUPPORTING INFORMATION}

Additional supporting information may be found in the online version of this article at http://onlinelibrary.wiley.com/doi/ 10.1002/ecy.3511/suppinfo

\section{OPEN RESEARCH}

All data (Liu et al. 2021) used for the analyses and figures contained in this manuscript are openly available in the Figshare digital repository: https://doi.org/10.6084/m9.figshare.14635374.v1 